\title{
Socialisme et sciences sociales : Sidney Webb et la création de la London School of Economics
}

Sidney Webb and the Creation of the London School of Economics

\section{Arnaud Page}

\section{(2) OpenEdition}

12 Journals

Édition électronique

URL : http://journals.openedition.org/rfcb/6136

ISSN : 2429-4373

Éditeur

CRECIB - Centre de recherche et d'études en civilisation britannique

Édition imprimée

Date de publication : 1 juin 2010

ISSN : 0248-9015

\section{Référence électronique}

Arnaud Page, « Socialisme et sciences sociales : Sidney Webb et la création de la London School of Economics ", Revue Française de Civilisation Britannique [En ligne], XV-4 | 2010, mis en ligne le 01 juin 2010, consulté le 07 janvier 2021. URL : http://journals.openedition.org/rfcb/6136

Ce document a été généré automatiquement le 7 janvier 2021.

\section{(†) $\odot$

Revue française de civilisation britannique est mis à disposition selon les termes de la licence Creative Commons Attribution - Pas d'Utilisation Commerciale - Pas de Modification 4.0 International. 


\title{
Socialisme et sciences sociales : Sidney Webb et la création de la London School of Economics
}

\author{
Sidney Webb and the Creation of the London School of Economics
}

\author{
Arnaud Page
}

1 Nous proposons d'aborder la question de l'inscription de la production du savoir dans un contexte matériel et politique à travers l'étude de la création de la London School of Economics par Sidney Webb en 1895. Le projet du leader socialiste et la création de cette institution soulèvent en effet de nombreuses questions relatives à l'objectivité et au caractère scientifique (ou non) de la représentation des phénomènes politiques, économiques et sociaux en Grande-Bretagne. Il est ainsi frappant, à première vue, de constater la façon dont Webb entreprit de présenter cette école comme un lieu de recherches impartial, sans aucune orientation politique ou partisane. Il convient cependant de revenir sur les variations et les ambiguïtés du projet de Webb, qui était initialement orienté principalement autour de la recherche et des préoccupations collectivistes, et qui fut réorienté progressivement vers la création d'une institution d'enseignement pluridisciplinaire de type universitaire. Nous tenterons ainsi de montrer que si le discours de Webb fut marqué de façon croissante par l'insistance sur le caractère « objectif » et « impartial » de la LSE, son projet était en réalité marqué par une conscience aiguë de l'inévitabilité des médiations politiques ou éthiques dans la découverte, la transmission et l'application politique des connaissances sur le monde social.

2 En décembre 1894, alors que la LSE n'était encore qu'un projet aux contours mouvants et peu définis, Beatrice Webb écrivait dans son journal: "Nous souhaitons introduire l'expert professionnel en politique, de façon à étendre le champ de l'action gouvernementale, en ajoutant à ses énormes avantages d'administration contraignante et à grande échelle, ceux des entrepreneurs les plus qualifiés $»^{1}$. Quelques mois plus tard, Beatrice Webb réaffirmait son insatisfaction vis-à-vis de la formation de la classe politique britannique et sa volonté de transformer la «profession» politique, qui devait selon elle devenir un domaine 
d'expertise "technique », comme l'ingénierie ou la médecine. Elle ajoutait que l'idéal de l'éducation "libérale» des anciennes universités devait être abandonné et qu'il s'agissait désormais de former des professionnels et des experts : «Nous ne voulons pas que les grandes fonctions gouvernementales soient occupées par des écoliers doués (clever schoolboys). Nous voulons des adultes, formés pour la tâche particulière dont ils sont chargés, [...] et se comportant à l'égard de leur profession comme le grand ingénieur civil, l'avocat ou le médecin $»^{2}$.

Dix ans plus tard, le 7 juillet 1905, Lord Rothschild (en tant que Président honorifique de la LSE), Halford Mackinder (directeur de la LSE de 1903 à 1908) et Sidney Webb adressaient une lettre à Austen Chamberlain, chancelier de l'Échiquier et responsable de la répartition des financements publics accordés aux établissements d'enseignement supérieur. Les auteurs de cette lettre y demandaient un renouvellement de l'aide gouvernementale accordée à la LSE et prenaient à cet égard soin de distinguer la LSE des institutions dispensant un savoir jugé « technique » ou « professionnel » (écoles de médecine, d'ingénieurs et d'agriculture) qui ne bénéficiaient pas de la dotation. Ils insistaient au contraire sur la parenté de la LSE et des colleges de l'Université (University, King's et Bedford) et sur le fait que l'École était « un lieu d'enseignement général, et non spécialisé". Ils ajoutaient enfin que "dans la faculté de sciences économiques et politiques, l'enseignement délivré à l'École est principalement du type libéral, et [...] même dans le cas des enseignements professionnels, [...] c'est la dimension libérale plutôt que technique qui [est] mise en avant. " ${ }^{3}$ 'écart entre les propos de Beatrice Webb et le contenu de cette lettre s'explique avant tout par la nature tout à fait différente des deux documents (l'un est privé, l'autre est collectif, officiel et destiné à un ministre, l'un dessine un projet politique, l'autre est avant tout mû par des considérations financières). La juxtaposition de ces deux documents semble néanmoins indiquer la nécessité de tenir compte de la multiplicité des discours sur l'École, de réinscrire ceux-ci dans leurs contextes respectifs et de mettre en lumière leurs variations et leurs différentes motivations.

\section{Un projet néopositiviste ${ }^{4}$ ?}

De toutes les institutions créées ou dirigées par les Webb, la LSE semble être, à première vue, celle qui était le plus directement fondée sur un projet "scientiste", au service d'un programme d'ingénierie sociale reposant non pas sur des considérations éthiques à propos du lien ou de la justice sociale, mais sur la notion "d'efficacité " ${ }^{5}$. Mark Bevir affirme d'ailleurs que le collectivisme de Webb n'est que la conséquence seconde d'un engagement plus constant et plus profond en faveur de l'organisation scientifique et

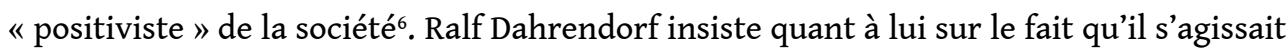
de former à la LSE « une nouvelle caste d'experts administratifs, l'avant-garde, pour ne pas dire la nomenklatura de l'État Positiviste ${ }^{7}$. On trouve en effet dans les journaux de Beatrice Webb, à l'époque de la création de la LSE, de multiples références à l'application de "la méthode scientifique aux questions sociales $»^{8}$. Beatrice fait part dans ces pages de sa volonté de s'attacher les services d'un groupe de jeunes étudiants intéressés par l'économie, « de libérer leurs esprits des préjugés » et de trouver ainsi, grâce aux « vrais faits » la solution de chaque "problème particulier », au-delà de tout biais ou orientation théorique et politique?.

5 S'il s'agissait effectivement de développer l'étude des phénomènes sociaux et de rendre le processus politique plus efficace grâce à ces découvertes, la position des Webb n'en 
conservait pas moins une forte orientation politique collectiviste. Leur foi en la science cohabitait avec une conscience très aiguë des problèmes relatifs à la fiabilité du matériau primaire et à sa manipulation théorique et idéologique. Il convient d'ailleurs de rappeler que la création de la LSE fut initialement conçue comme une stratégie de remplacement à la suite d'une défaite politique :

Notre tentative d'assurer une majorité permanente à un parti progressiste à Londres ayant échoué, nous nous occupons désormais de la création de la London School of Economics and Political Science [...]. L'École est réellement scientifique, la plupart des enseignants n'appartiennent d'ailleurs pas au camp collectiviste, les individualistes demeurant pour l'heure les plus compétents. Mais les collectivistes y sont encouragés, et les jeunes hommes et femmes sont soumis à l'influence collectiviste. Nous essayons dans une certaine mesure de rassembler les étudiants prometteurs autour de nous. ${ }^{10}$

6 On note ainsi que l'affirmation selon laquelle l'École était « réellement scientifique » ne s'accompagnait en aucun cas d'une ignorance de la présence de médiations politiques dans la production du savoir, Beatrice soulignant au contraire les différentes orientations idéologiques des enseignants. Quelle qu'ait été la foi des Webb dans la possibilité d'étudier objectivement les « faits » sociaux puis d'importer le fruit de cette connaissance objective dans le champ administratif, Beatrice continuait en réalité de caresser l'ambition de voir la LSE devenir «un centre de travail et de camaraderie intellectuels à partir duquel nos opinions rayonneront grâce aux rapports personnels » et ainsi promouvoir non seulement la science mais également les idées collectivistes ${ }^{11}$.

7 En dépit d'un projet en apparence destiné à promouvoir la production de connaissances «techniques » sur le monde social, l'essentiel du travail développé à la LSE demeura ainsi marqué par la persistance d'une approche ouvertement politique et réformiste, sous-tendue par un impératif éthique et rejetant toute utopie fondé sur le scientisme ou l'expertocratie. L. T. Hobhouse, Graham Wallas et la plupart des principaux enseignants de la LSE (Edwin Cannan, A. L. Bowley) étaient tous des universitaires formés à Oxford et Cambridge et leur attachement conjoint à la démocratisation et au développement des connaissances sur le monde social s'accompagnait d'une croyance en la nécessité d'une nouvelle forme de moralité ${ }^{12}$. Il faudrait d'ailleurs nuancer le "positivisme " (à la fois en termes de succession d'états historiques et de promotion d'une société organisée de façon plus scientifique) des Webb eux-mêmes dont l'approche intellectuelle et politique était en réalité tout à fait conciliable avec la persistance d'une approche morale et de discours extra-scientifiques ${ }^{13}$. S'il existait selon eux une loi historique inhérente aux sociétés industrielles modernes, tendant à guider celles-ci vers un mode d'organisation plus collectiviste, ces lois historiques étaient accompagnées par un impératif éthique. Beatrice Webb évoquait ainsi fréquemment, dans son journal, la nécessité d'une nouvelle forme sécularisée de moralité et l'incapacité de la science à remplacer les valeurs philosophiques ou éthiques, insistant ainsi sur «la faillite de la science lorsqu'elle tente de rendre compte de la cause ou du but de l'existence humaine $»^{14}$.

\section{Un héritage inattendu}

8 Au-delà des idées exprimées par Beatrice Webb dans ses journaux, il convient de bien prendre en compte la variation du projet pour l'École et du discours sur celle-ci, qui s'inscrivent dans une large mesure dans une dimension pragmatique de légitimation. 
La création de la London School of Economics and Political Science est directement liée au décès d'un riche avocat de Derby et membre de la Fabian Society, Henry Hunt Hutchinson, qui se suicida le 26 juillet 1894. Dans son testament, rédigé en octobre de l'année précédente, il disait léguer sa fortune non pas directement à la Fabian Society, mais à Sidney Webb, William Clarke, William S. De Mattos et Edward R. Pease, tous membres de la Société. Ceux-ci devaient administrer sa fortune et la consacrer, « de la façon qu'ils jugeraient souhaitable ", "à la propagande et aux autres objectifs » de "la Fabian Society et de son socialisme $»^{15}$. Ayant été informé de la mort de Hutchinson (qu'il ne connaissait pas) et du contenu de son testament, Webb écrivit à Pease le 3 août 1894 pour l'en informer, et surtout pour lui enjoindre de n'en parler à personne, afin de ne pas attiser les convoitises. Il semble notamment que le notaire de Hutchinson doutait de la légalité du testament, dont les termes étaient extrêmement vagues et dont on pouvait craindre qu'il n'ait pas été rédigé par une personne en pleine possession de ses moyens ${ }^{16}$. Si le récit traditionnel sur la création de la LSE veut que Webb ait informé Graham Wallas et George Bernard Shaw de ses projets d'école de sciences sociales lors d'un petit déjeuner le jour suivant ( 4 août $)^{17}$, la première mention écrite de la création d'une école de sciences politiques intervient en réalité dans une entrée du journal de Beatrice Webb, datée du 21 septembre 1894 :

La simple propagande doctrinaire en faveur du collectivisme se développe rapidement à travers le ILP; il semblerait que la majorité des ouvriers seront devenus collectivistes avant la fin du siècle. Mais ce ne sont pas ces cris qui permettront la mise en place de réformes. Ce dont nous avons avant tout besoin, c'est d'une solide réflexion. La même objection s'applique d'ailleurs à l'envoi de socialistes insignifiants au Parlement. Les députés radicaux sont assez ouverts et accommodants : ce qui leur manque, c'est le levain de la connaissance. Sidney a donc entrepris de persuader les autres administrateurs de consacrer la majeure partie de l'argent à l'encouragement de la recherche et de l'étude de l'économie. Son objectif est de fonder, lentement et sans éclats, une « London School of Economics and Political Science ", un centre de cours sur des sujets particuliers, mais également une association d'étudiants qui seraient supervisés et encouragés à faire des recherches..$^{18}$

9 Si le nom de "London School of Economics and Political Science " fut ainsi employé par Beatrice dès septembre 1894, il ne semble pas que ceci fut accompagné de l'élaboration d'un projet cohérent, et encore moins de la volonté de créer un centre de sciences sociales de type universitaire. Il est surtout intéressant de noter qu'à aucun moment dans la correspondance de la fin de l'année 1894 entre Webb et Pease (seul membre du Hutchinson Trust auquel Webb faisait part de ses projets), il n'est fait mention d'un projet ressemblant à ce qui allait devenir la LSE $^{19}$. À en juger par cette correspondance, la préoccupation principale de Webb était que l'argent de Hutchinson ne soit pas réclamé par sa veuve, ce dont il s'assura en doublant la maigre somme que lui avait accordée son époux. La première réunion des administrateurs, le 26 septembre 1894, fut ainsi exclusivement consacrée à cette question sans qu'il soit fait mention de projets

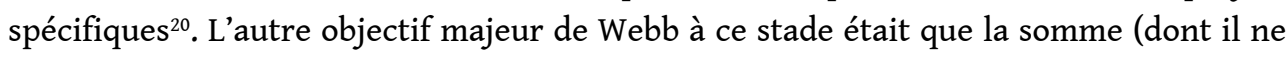
connaissait pas encore l'importance) demeurât aux mains des Hutchinson Trustees (c'està-dire dans les siennes) et ne fût accaparée par la Fabian Society, qu'il contrôlait moins. Webb était tout à fait opposé à ce que l'argent de Hutchinson soit utilisé pour financer les actions traditionnelles de la Société (tenue de conférences et publication de brochures), ce qui aurait eu selon lui comme conséquence une diminution des contributions et dons à la sociétée ${ }^{21}$. En revanche, Webb ne précisait pas comment il 
comptait dépenser de cet argent si ce n'est qu'il s'agissait bien d'encourager un travail plus "solide" de recherche afin de développer les fondations intellectuelles sur lesquelles reposaient les activités traditionnelles de la Fabian Society.

Tout en s'employant à contrôler la totalité de l'argent de Hutchinson, Webb entreprit de prévenir toute opposition de la part de la Fabian Society, et envoya une lettre aux membres les plus importants de la Société afin que ceux-ci lui soumettent des suggestions quant à la meilleure façon de dépenser cette somme importante (environ $€$ 9000 ). Il reçut huit réponses, proposant notamment la création d'un journal de la Fabian Society, d'une école de «fabianisme " par correspondance ou encore d'un centre d'études consacré aux questions internationales ${ }^{22}$. S'il ne fait aucun doute que le discours tenu par Webb auprès des autres Fabiens devait nécessairement insister sur la dimension proprement politique du projet qui serait mis en place, il est intéressant de constater que les propos de Webb, à la fin de l'année 1894, étaient constamment articulés autour des besoins du mouvement socialiste et ne dissociaient jamais les plans scientifique et politique de son projet. De façon tout à fait caractéristique, Webb demandait aux membres de la Fabian Society leur avis, tout en entreprenant avant tout d'imposer le sien et d'insister sur la façon dont cet argent devait être dépensé :

Les besoins les plus immédiats du mouvement collectiviste en Angleterre à l'heure actuelle semblent être (1) une plus large diffusion auprès de la population et en particulier auprès des socialistes et des membres de l'ILP de connaissances exactes sur la dimension économique de l'administration publique ; (2) des recherches plus approfondies sur les questions non résolues relatives au gouvernement national et municipal, de façon à renouveler et rafraîchir la propagande ; (3) la nécessité d'attirer des recrues intelligentes et éduquées pour étudier ces questions. ${ }^{23}$

11 Afin de s'assurer du soutien de la Fabian Society, une partie de l'argent de Hutchinson fut ainsi dévolue à la mise en place d'un cycle de conférences, de nature plus politique, de la Fabian Society à travers le pays ${ }^{24}$. Il est en revanche à noter que Ramsay MacDonald avait également sollicité l'aide financière des Hutchinson trustees, en vue de fonder une revue, la Progressive Review, mais il fut décidé de ne pas accéder à cette requête ${ }^{25}$.

La décision de créer la London School of Economics and Political Science fut en réalité officiellement prise le 8 février 1895. Quelques jours plus tôt, Webb avait rédigé un courrier à l'attention des Hutchinson Trustees, dans lequel il insistait sur le fait que leur objectif principal devait être «l'éducation" plutôt que la "propagande». Il entreprit ainsi de présenter son projet d'une London School of Economics and Political Science, dont l'esprit devait être "scientifique et non partisan ». On notera néanmoins que le discours de Webb continuait d'être articulé autour des «besoins du mouvement collectiviste en Angleterre ", de l'approfondissement des connaissances sur l'administration municipale et nationale "du point de vue collectiviste " et de la nécessité d'attirer des recrues qui soient compétentes et favorablement disposées à l'égard des idées socialistes ${ }^{26}$. Dans ce même document, Webb avait dressé une liste des thèmes qui devaient être étudiés dans cette nouvelle institution, l'accent étant pour l'instant mis avant tout sur la recherche, l'enseignement étant clairement subordonné à l'impératif de production de connaissances nouvelles. Cette liste, constituée de l'aveu de Webb avant tout en fonction des personnes de son entourage susceptibles de conduire ce type de travail, montre là encore que l'objectif principal, dans ce premier projet, était le développement de connaissances précises et les plus objectives possibles mais toujours en lien direct avec les idées socialistes : 
Les sujets qui me sont apparus comme ceux pour lesquels nous pourrions commencer immédiatement et faire du bon travail sont les suivants. (Choisis avant tout parce que je connais des hommes disponibles qui feraient l'affaire) :

Histoire de la régulation des salaires par la loi, et ses résultats.

Essor et développement du mouvement ouvrier anglais (Chartisme, etc.).

Rouages de la démocratie (en Grande-Bretagne et à l'étranger).

Arbitrage et conciliation, échelles mobiles, etc.

Économie des chemins de fer.

Expérimentations dans le domaine de la législation industrielle. ${ }^{27}$

13 On remarquera ainsi qu'hormis le thème spécialisé des chemins de fer (en partie destiné à attirer un nombre important d'étudiants de façon à pouvoir financer les autres activités de l'école) et un domaine d'étude très général («rouages de la démocratie »), les autres thèmes proposés par Webb démontrent très clairement une volonté d'accumuler des connaissances très proches des préoccupations collectivistes, qu'il s'agisse de l'histoire du mouvement ouvrier anglais, de celle la régulation de la vie économique et industrielle par des instances politiques ou de l'étude des différents types d'interférences de l'État dans l'économie.

Il est en outre important de noter que si c'est finalement l'économiste d'Oxford W.A.S. Hewins qui devint le premier directeur de l'École, Webb avait d'abord proposé ce poste à un fabien, Graham Wallas, qui l'avait refusé au mois de mars $1895^{28}$. Hewins n'était pas membre de la Fabian Society et ne pouvait en aucun cas être considéré comme un socialiste. Il avait suivi des études de mathématiques et d'économie à Oxford, et il enseignait, en 1895, l'économie au sein du mouvement d'University Extension, tout en étant tuteur en histoire économique à Pembroke College. Les Webb savaient qu'il travaillait dans le domaine de l'histoire économique mais le connaissaient très peu, l'ayant seulement rencontré de façon fortuite à la Bodleian Library. On notera néanmoins que même après la nomination de Hewins, l'ébauche de liste de cours continua pendant quelque temps d'être marquée par une orientation en faveur des problématiques touchant directement le mouvement travailliste et collectiviste puisqu'il s'agissait avant tout de s'intéresser à "l'État en lien avec le commerce et l'industrie » et à "l'arbitrage et la conciliation » des conflits sociaux ${ }^{29}$. Si Hewins devint député conservateur par la suite, il ne faudrait d'ailleurs pas surestimer les divergences de point de vue, à l'époque, entre Webb et Hewins, pour qui la tâche première des sciences sociales modernes était de conduire des recherches empiriques permettant de mettre à jour la complexité des liens sociaux unissant les individus et ainsi de rejeter le concept d'" homme économique " (une " abstraction ricardienne " selon Hewins) ${ }^{30}$. Si Webb avait initialement pensé à Wallas pour le poste de Directeur, le choix de Hewins s'avérait en réalité doublement intéressant puisqu'il permettait de confier l'École à quelqu'un dont les idées scientifiques et politiques n'étaient - à cette époque - pas très éloignées des siennes, mais qui pouvait en même temps être présenté comme un adversaire politique, légitimant ainsi le discours sur « l'impartialité » de l'École.

\section{Discours de légitimation}

Dans les mois qui suivirent le projet de la LSE fut en effet réorienté de façon importante, les discours de Hewins et Webb étant marqués de façon croissante par l'affirmation de la neutralité politique de l'École, de façon à s'assurer le soutien financier d'institutions comme la Chambre de Commerce de Londres, la Society of Arts 
ou le Technical Education Board (TEB) du London County Council (LCC). La réputation de Webb n'était évidemment pas sans susciter de vives inquiétudes quant à l'orientation idéologique de l'École, et la Chambre de Commerce stipula que son aide dépendait de la neutralité politique des enseignements délivrés à la LSE, et certains cours envisagés initialement furent même supprimés ou modifiés ${ }^{31}$. Hewins fut également sommé par la Society of Arts, dont il avait entrepris d'obtenir le soutien, de donner des gages de la neutralité politique de l'École ${ }^{32}$. Ajoutons qu'à partir de la nomination de Hewins, Webb ne se consacra pratiquement plus aux aspects académiques et au contenu des enseignements délivrés par l'École. Sa préoccupation principale était d'assurer le développement de l'institution, sa solvabilité financière, le soutien d'autant d'institutions que possible et son incorporation dans la nouvelle Université de Londres. Les nombreuses activités de Webb au sein de la LSE relevaient ainsi toutes de l'aspect financier et administratif, et l'on ne trouve pratiquement aucune trace d'intervention de Webb dans le domaine académique. Il est ainsi intéressant de constater le partage des tâches qui fut opéré entre Webb et Hewins, qui se chargea de recruter la plupart des professeurs et de prendre toutes les décisions relatives à l'organisation de l'enseignement. Il n'est ainsi pas inintéressant de noter que sous l'influence de Hewins, universitaire formé à Oxford, la LSE prit un tournant plus académique, en accordant davantage d'importance à l'enseignement et en se présentant comme une institution neutre politiquement. Ainsi, si les sujets d'études évoqués par Webb quelques mois plus tôt avaient été relativement spécialisés, orientés politiquement, et avaient été mentionnés avant tout en fonction des personnes que Webb connaissait pour les superviser, le programme d'enseignement établi par Hewins était beaucoup plus systématique et organisé autour des aspects fondamentaux de disciplines (économie, statistiques, finances, etc.), sans doute plus à même d'attirer des étudiants. Dans le premier prospectus publié par la London School of Economics quelque temps avant l'ouverture effective de l'École, on constate ainsi que le discours sur la nature de cette institution avait profondément évolué. Non seulement la question du collectivisme avait-elle dans une large mesure disparu mais la LSE était à présent dépeinte comme un lieu d'enseignement tout autant sinon plus que comme un centre de recherche. L'École ouvrit ses portes le 10 octobre 1895 et accueillit, lors du premier trimestre, environ 200 étudiants qui pouvaient y suivre des cours d'économie, de science politique, de finances publiques, de statistiques, de finance, de droit commercial, d'histoire et de géographie commerciale $^{33}$.

$\mathrm{Au}$ cours des vingt premières années d'existence de la LSE, Webb fut fréquemment sommé de réaffirmer l'indépendance politique de la LSE afin d'assurer la survie financière de cet établissement. La position de la LSE dans ses premières années d'existence demeurait extrêmement précaire, et l'objectif premier de Webb comme de Hewins (jusqu'au débat sur le protectionnisme de 1903) était de ménager autant de soutiens financiers et institutionnels que possible pour l'École. Ainsi, en 1898, lorsque Edward Pease proposa la nomination d'un membre de la Fabian Society (Frederik Whelen) comme membre du comité de direction de l'École (Administrative Committee), Hewins s'opposa très fermement à cette nomination, arguant qu'il n'avait aucune " objection à la nomination d'un partisan radical, tant qu'il [était] suffisamment reconnu. [...] Peut-être serait-il plus sage dans les conditions actuelles de consulter [R. B.] Haldane avant toute nouvelle nomination, de façon à ce que toute nouvelle personne renforce la position de l'École visà-vis des membres de la commission [pour l'Université de Londres]. " ${ }^{34} \mathrm{En}$ sa qualité de directeur du Technical Education Board du London County Council (qui finançait la LSE), 
Webb était, plus que Hewins, contraint de dissocier ses fonctions au TEB et à la LSE pour ne pas alimenter les critiques et les accusations de conflit d'intérêts que son omniprésence au sein de ces diverses institutions avait fait naître. Lors de la controverse à propos du protectionnisme (1903), il se montra très préoccupé par la participation active de Hewins à ce débat politique, dont les conséquences ne pouvaient selon Webb être que néfastes pour l'École. Il fit part de ses inquiétudes à Hewins, lui demandant de respecter une certaine réserve afin de protéger l'impartialité de l'École ${ }^{35}$. Celui-ci n'en fit rien, et finit par démissionner de ses fonctions de directeur de l'École en novembre 1903 afin de devenir secrétaire de la "Commission sur les droits de douane " (Tariff Commission) de Joseph Chamberlain. Hewins essaya ensuite de devenir Membre du Parlement, échouant à trois reprises avant d'être élu à Hereford City en 1912 comme membre du Parti conservateur (jusqu'en 1918) ${ }^{36}$.

De façon plus générale, Webb se montra de plus en plus insistant sur la question de la neutralité politique afin notamment de légitimer l'incorporation de la LSE à l'Université de Londres. La quête de respectabilité académique le poussa par exemple, pour infirmer toute accusation de collusion entre la LSE et la Fabian Society, à nier le rôle moteur du legs de Hutchinson dans la création de l'École. A la suite de nouvelles attaques concernant la légitimité de l'institution, Webb adressa en effet, en 1903, une lettre au vice président de l'Université de Londres, dans laquelle il affirmait que la LSE avait été soutenue financièrement par de multiples institutions et donateurs, le Hutchinson Trust ne représentant ainsi qu'une source de revenus parmi d'autres, et sans qu'aucune donation ait donné lieu à l'imposition de conditions politiques ou doctrinaires. Afin de renforcer la légitimité de cette version, et de montrer que la création de la LSE avait été précédée par la lente maturation d'un projet intellectuel cohérent, Webb insista en outre sur la proximité entre ses préoccupations et les inquiétudes sur l'état des sciences sociales en Grande-Bretagne exprimées quelques temps avant la création de la LSE par les membres de la commission chargée de créer la nouvelle Université de Londres ainsi que par la British Association ${ }^{37}$.

\section{Conclusions}

18 En 1914, 20 ans après la mort de Hutchinson, la LSE était devenue une institution solidement établie, l'une des plus importantes composantes de l'Université de Londres, et le seul établissement du pays consacré uniquement aux sciences sociales. L'argent de Hutchinson avait été dépensé depuis plus de 10 ans, et l'École avait pris une ampleur considérable. Elle accueillait environ 2000 étudiants par an, dont près de 400 graduate students. Elle décernait environ $30 \mathrm{BSc}$ par an et une vingtaine d'étudiants avaient obtenu le titre de Doctor of Science (Econ.) depuis la création de l'institution. Plus de 200 cours différents y étaient dispensés, du plus technique au plus théorique et sa bibliothèque contenait plus de 300000 documents. Ce développement important s'était néanmoins fait en partie au prix d'une modification du projet initial. Webb envisageait à l'origine de constituer un organisme de recherche manifestement destiné à devenir un instrument au service des idées collectivistes, et était parfaitement conscient des implications politiques de son projet. La référence au collectivisme disparut néanmoins progressivement et l'insistance, initialement présente mais non unique, sur le caractère neutre et impartial de l'École devint de plus en plus importante, de façon à assurer financement et respectabilité académique à une institution universitaire 
d'enseignement supérieur ${ }^{38}$. Loin de procéder d'une vision naïvement positiviste, l'affirmation du caractère non partisan de cette institution et des connaissances qui y étaient produites était ainsi, dans une large mesure, le fruit de considérations tactiques. La foi indéniable des Webb en la possibilité d'améliorer les processus politiques grâce à l'expertise n'était ainsi pas incompatible avec une conscience aiguë du caractère problématique de la fiabilité et des possibilités de manipulation de toute représentation de la société britannique, phénomènes qui devaient néanmoins être fréquemment minimisés ou passés sous silence pour des raisons pragmatiques de consolidation institutionnelle.

\section{NOTES}

1. 'We wish to introduce into politics the professional expert - to extend the sphere of government by adding to its enormous advantages of wholesale and compulsory management, the advantage of the most skilled entrepreneur.' Journal de Beatrice Webb, 28 décembre 1894, in Norman \& Jeanne MACKENZIE (eds.), The Diary of Beatrice Webb: Volume Two, 1892-1905, Cambridge (Mass.): Belknap, 1983, p. 63.

2. 'We do not want clever schoolboys at the head of our great departments. We want grown men - "grown up" in the particular business they take in hand, [...] behaving towards their profession as the great civil engineer, lawyer, or medical man behaves.' Journal de Beatrice Webb, 20 juin 1896, in ibid., p. 97.

3. '[...] In the Faculty of Economics and Political Science, the teaching of the School is, in the main, of the liberal type for which the grant in aid is voted. [...] Even in the case of the Professional subjects, which are analogous to the Medical and Technical subjects at other University Colleges, it is the liberal rather than the practical side which is emphasised [...].' Lettre de Lord Rothschild, Sidney Webb \& Halford Mackinder à Austen Chamberlain, chancelier de l'Échiquier, 7 juillet 1905, Administrative Committee, LSE Archives.

4. Le « néopositivisme » dont la LSE est supposée être l'unique représentante en Grande-Bretagne au début du vingtième siècle est caractérisé comme reposant sur une méthodologie strictement empiriste et sur l'idée que la science est la seule source légitime de connaissance, en dehors de toute considération religieuse ou métaphysique. (Dorothy ROSS, 'Changing Contours of the Social Science Disciplines', in Dorothy ROSS \& Theodore PORTER (eds.), The Cambridge History of Science, vol. 7, The Modern Social Sciences, Cambridge: Cambridge University Press, 2003, pp. 214-215). Aucun de ces aspects ne correspond selon nous à la réalité des vingt première années d'existence de la LSE.

5. G. R. SEARLE, The Quest for National Efficiency: A Study in British Politics and Political Thought, 1899-1914, Londres: The Ashfield Press, 1990 (1971), pp. 84 \& 124-125; E. J. T. BRENNAN (ed.), Education for National Efficiency: The Contribution of Sidney and Beatrice Webb, Londres: the Athlone Press, 1975, pp. 41-44. Il ne nous est pas possible d'évoquer de façon précise dans cet article les liens entre la LSE et le mouvement de National Efficiency des années 1900. On pourra à ce sujet se référer à l'ouvrage récent de Jennifer Karns Alexander qui ne traite pas de ce mouvement politique, mais montre justement que la notion d'efficacité n'est pas propre au début du vingtième siècle, et qu'il faut moins la voir comme une idéologie précise que comme un vocable fondamental de la modernité, utilisé à ce titre pour justifier des programmes administratifs ou 
politiques fort différents (Jennifer Karns ALEXANDER, The Mantra of Efficiency: From Waterwheel to Social Control, Baltimore: John Hopkins University Press, 2008).

6. Mark BEVIR, 'Sidney Webb: Utilitarianism, Positivism and Social Democracy', Journal of Modern History, vol. 74, $\mathrm{n}^{\circ}$ 2, juin 2002, pp. 217-25.

7. '[According to the Fabians], a caste of experts had to be trained; the vanguard, not to say the nomenklatura of the Positivist State.' Ralf DAHRENDORF, LSE: A History of the London School of Economics, 1895-1995, Oxford: Oxford University Press, 1995, p. 42.

8. Beatrice Webb à Charlotte Payne-Townshend, 28 juin 1896, in Norman \& Jeanne MACKENZIE (eds.), The Letters of Sidney and Beatrice Webb: Volume II, Partnership, 1892-1912, Cambridge: Cambridge University Press, 1978, p. 47.

9. 'I should like to gather round us all the able young men and women who are taking to economics, free their minds of prejudices and start them with a high ideal of accuracy and exhaustiveness in work. If there is one thing I have believed 'from the beginning to the end', it is that no progress can be made except on the basis of ascertained fact and carefully thought-out suggestion. Despite our theory, bias, creed and prejudice, we are all equally wandering in the labyrinth, searching for the clue of true facts to bring us out on the right side of each particular problem.' Journal de Beatrice Webb, 8 mai 1895, in Norman \& Jeanne MACKENZIE (eds.), The Diary of Beatrice Webb: Volume Two, 1892-1905, op. cit., p. 73.

10. 'Having been beaten back in our endeavour to make a London Progressive Party with a permanent majority, we are creating the London School of Economics and Political Science [...]. It is honestly scientific, served indeed by more individualist lecturers than collectivists, because the individualists are still the better men. But collectivists are encouraged, and the younger men and women are brought under “collectivist" influence.' Journal de Beatrice Webb, 25 décembre 1895, in Norman \& Jeanne MACKENZIE (eds.), The Diary of Beatrice Webb: Volume Two, 1892-1905, op. cit., p. 85.

11. 'We want to create a centre of intellectual work and comradeship from which our views will radiate through personal intercourse.' Journal de Beatrice Webb, 16 septembre 1896, in Norman \& Jeanne MACKENZIE (eds.), The Diary of Beatrice Webb: Volume Two, 1892-1905, op. cit., p. 100.

12. Il est tout à fait clair que les Webb se réjouirent, lors de l'incorporation de la LSE à l'Université de Londres, du fait que l'économie soit considérée comme une science et non comme une «humanité ». Beatrice se félicitait ainsi que le diplôme couronnant les études menées à la LSE soit un BSc et non un BA. Journal de Beatrice Webb, 20 février 1900, in Norman \& Jeanne MACKENZIE (eds.), The Diary of Beatrice Webb: Volume Two, 1892-1905, op. cit., p. 170. A titre anecdotique on pourrait néanmoins souligner que l'importance accordée par les Webb aux atours symboliques de la 'scientificité' n'était pas univoque. En dépit du fait que Webb avait été, par exemple, très impressionné lors de sa visite au MIT en 1888, il insista 11 ans plus tard, contre l'avis du principal donateur, pour que le bâtiment central de la LSE fût nommé 'Hall' et non 'Institute', au titre que « Oxford, Cambridge et la plupart des autres universités [étaient] constituées par des 'Halls' et non des 'instituts'». Sidney Webb à John Passmore Edwards, 17 août 1899, Passfield Papers.

13. Sur ce point, voir José HARRIS, 'The Webbs, the Charity Organisation Society and the Ratan Tata Foundation: Social Policy from the Perspective of 1912', pp. 27-63 in Martin BULMER et al., The Goals of Social Policy, Londres: Unwin Hyman, 1989.

14. A l'occasion de la mort de Herbert Spencer, Beatrice écrivait ainsi : 'His failure to attain to the higher levels of conduct and feeling has sealed my conviction in the bankruptcy of science when it attempts to realize the cause or the aim of human existence.' Journal de Beatrice Webb, 9 décembre 1903, in Norman \& Jeanne MACKENZIE (eds.), The Diary of Beatrice Webb: Volume Two, 1892-1905, op. cit., p. 308.

15. 'The residue of my property I bequeath in trust and jointly to said Constance [Hutchinson] and Webb together with his colleagues of [the Fabian Society] William H. De Mattos, William Clarke and Edward R. Pease, that they may apply the same at once, gradually and at all events within ten years to the propaganda and other purposes of the said society and its socialism, and towards advancing its objects in 
any way they deem advisable.' Testament de Henry Hunt Hutchinson, Hutchinson Papers, LSE Archives.

16. Sidney Webb à Edward Pease, 3 août 1894, cité in Norman \& Jeanne MACKENZIE (eds.), The Letters of Sidney and Beatrice Webb: Volume II, Partnership, 1892-1912, Cambridge: Cambridge University Press, 1978, p. 21.

17. Graham WALLAS, 'An Historical Note', The Students' Union Handboook, 1925, in LSE History / Box 39 I ; Sidney \& Beatrice WEBB, 'Reminiscences, IV: The London School of Economics and Political Science', St Martin's Review, janvier 1929, pp. 24-25 ; Janet BEVERIDGE, An Epic of Clare Market, Londres: G. Bell \& Sons, 1960, pp. 18-19 ; Ralf DAHRENDORF, A History of the London School of Economics and Political Science, 1895-1995, Oxford: Oxford University Press, 1995, pp. 4-5.

18. 'Mere propaganda of the shibboleths of collectivism is going on at a rapid rate through the ILP; [...] It looks as if the great bulk of the working-men will be collectivists before the end of the century. But reform will not be brought about by shouting. What is needed is hard thinking. And the same objection applies to sending nondescript socialists into Parliament. The Radical members are quite sufficiently compliant in their views: what is lacking in them is the leaven of knowledge. So Sidney has been planning to persuade the other trustees to devote the greater part of the money to encouraging research and economic study. His vision is to found, slowly and quietly, a "London School of Economics and Political Science" - a centre not only of lectures on special subjects, but an association of students who would be directed and supported in doing original work.' Journal de Beatrice Webb, 21 septembre 1894, in Norman \& Jeanne MACKENZIE (eds.), The Diary of Beatrice Webb: Volume Two, 1892-1905, Cambridge (Mass.): Belknap, 1983, p. 56.

19. Les comptes rendus des réunions indiquent que Constance Hutchinson, fille de Hutchinson, et co-administratrice avec Webb, ne participait pas aux réunions, pas plus que William Clarke. William De Mattos participa aux premières réunions, mais cessa assez rapidement de s'y rendre. Le Henry Hutchinson Trust était ainsi, en pratique, dirigé par deux personnes, Sidney Webb et Edward Pease.

20. Compte rendu de la réunion des Hutchinson Trustees, 26 septembre 1894, Hutchinson Papers.

21. Sidney Webb à Edward Pease, 25 août 1894, in Norman \& Jeanne MACKENZIE (eds.), The Letters of Sidney and Beatrice Webb: Volume II, op. cit., p. 22.

22. Norman \& Jeanne MACKENZIE (eds.), The Letters of Sidney and Beatrice Webb: Volume II, op. cit., p. 27.

23. 'The most pressing needs of the Collectivist movement in England at the present moment appear to be (1) a wider diffusion among the people and especially among Socialists and ILP members of accurate knowledge as to the economic bearings of public administration; (2) further research into the unsolved problems of municipal and national government, so as to renew and refresh the propaganda; (3) the attraction to these studies of clever and educated recruits.' Sidney Webb, 27 novembre 1894, Hutchinson Papers.

24. Il fut décidé d'accorder $€ 200$ à la Fabian Society, à dépenser dans l'année. Compte rendu de la réunion des Hutchinson Trustees, 28 mars 1895, Hutchinson Papers.

25. Compte rendu de la réunion des Hutchinson Trustees, 28 mars 1895, Hutchinson Papers. La Progressive Review fut néanmoins créée en octobre 1896, avec MacDonald pour secrétaire, mais sa publication dura moins d'un an. Kevin MORGAN, Ramsay MacDonald, Londres: Haus, 2006, p. 13.

26. Sidney Webb aux Hutchinson Trustees, 8 février 1895, Hutchinson Papers.

27. 'The subjects I thought about as such as we could take up at once and get good work done are the following. (Chosen rather because I know of suitable men available): The History of the Regulation of Wages by Law, and its results. Growth and development of English Working Class movement (Chartism, etc.). The Working of Democratic Machinery (home and foreign). Arbitration and Conciliation, Sliding Scales, etc. Railway Economics. Factory Act experiments.' Sidney Webb aux Hutchinson Trustees, 8 février 1895, Hutchinson Papers.

28. Compte-rendu de la réunion des Hutchinson Trustees, 28 mars 1895, Hutchinson Papers. 
29. 'The State in Relation to Commerce and Industry', 'Arbitration and Conciliation', avril 1895, Hutchinson Papers.

30. Il est d'ailleurs intéressant de constater que le rejet de la méthode abstraite et déductive par Hewins se fondait avant tout sur une volonté de nier le caractère proprement «scientifique » de l'économie et sur la nécessité de réintroduire les considérations éthiques dans les décisions politiques. W.A.S. HEWINS, 'Political Economy and the Scientific Method' (non publié, non daté), Hewins Papers, Articles and Prints / LSE Foundation, 419-443; W.A.S. HEWINS, 'The Teaching of Economics', International Congress on Technical Education: Report of the Proceedings of the fourth meeting, juin 1897, Hewins Papers, Articles and Prints.

31. 'Report of the Chamber of Commerce on the proposed evening lectures', 18 mai 1895, Hewins Papers, 14/68-70. Lettre du secrétaire de la Chambre de Commerce de Londres à W. A. S. Hewins, mai 1895, Hewins Papers, 43/197.

32. Henry Trueman Wood à Owen Roberts, cité in Ralf DAHRENDORF, A History of the London School of Economics and Political Science, 1895-1995, op. cit., p. 16.

33. W.A.S. HEWINS, The London School of Economics and Political Science, 1895, p. 2, Hutchinson Papers. 34. 'There can be no objections to an extreme partisan, so long as he is eminent enough. [...] Perhaps it would be wisest at the present juncture to consult Haldane before making any additions to the committee, so that any new man might strengthen the position of the School with the University Commissioners.' W.A.S. Hewins à Sidney Webb, 17 octobre 1898, Passfield Papers, 2 (i) 97. Le député Libéral R. B. Haldane, avec lequel les Webb collaboraient fréquemment, était l'un des principaux architectes de la création de la nouvelle Université de Londres (1898).

35. Sidney Webb à W.A.S. Hewins, 30 mai 1903, in Norman \& Jeanne MACKENZIE (eds.), The Letters of Sidney and Beatrice Webb: Volume II, op. cit., p. 22.

36. Ralf DAHRENDORF, A History of the London School of Economics and Political Science, 1895-1995, op. cit., p. 70 .

37. '[My wife and myself] had long been concerned at the lack of provision for (1) economic teaching and research, (2) training in administration, whether commercial or governmental.' Copie d'une lettre confidentielle de Sidney Webb au révérend Archibald Robertson, Vice-Chancellor de l'Université de Londres, 3 janvier 1903, in Norman \& Jeanne MACKENZIE (eds.), The Letters of Sidney and Beatrice Webb: Volume II, op. cit., p. 176.

38. Il s'agit bien entendu ici uniquement du discours sur l'École, les développements réels de celle-ci n'étant au contraire absolument pas marqués par une évacuation des questions directement politiques, comme on peut le constater notamment avec la forte polarisation de l'entre-deux-guerres entre les sciences politiques (Harold Laski) et l'économie (Lionel Robbins et Friedrich Hayek).

\section{RÉSUMÉS}

Cet article est consacré à une analyse du projet de Sidney Webb pour la London School of Economics (fondée en 1895). Il ne s'agit néanmoins pas d'étudier les propos de Webb comme diverses manifestations d'un projet idéologique homogène, mais avant tout comme des actions à replacer dans leurs contextes pragmatiques. Nous souhaiterions ainsi montrer que la création de cette École ne procède pas de la simple mise en œuvre d'un projet «néopositiviste " tout à fait cohérent, mais au contraire que le discours de Webb vis-à-vis de la question de l'objectivité était 
variable et répondait dans une large mesure à un objectif pragmatique de développement et de consolidation institutionnels.

This article analyses Sidney Webb's project for the London School of Economics, created in 1895. Rather than studying Webb's utterances as reflections of a homogenous ideological project, however, it attempts to analyze them as acts performed in different pragmatic contexts. This paper thus argues that the creation of the School was not the result of a simple and coherent 'neopositivistic' vision but that Webb's discourse on the question of objectivity must to an important extent be seen as a means to support his primary objective, the development and consolidation of the School.

\section{AUTEUR}

\section{ARNAUD PAGE}

Université de Pau et des Pays de l'Adour 